\title{
BMC Ophthalmology reviewer acknowledgement 2015
}

Anna Clark

\section{Contributing reviewers}

The editors of BMC Ophthalmology would like to thank all our reviewers who have contributed to the journal in Volume 15 (2015).

Mohammed Abdull

Nigeria

Massimo Accorinti

Italy

James Acheson

UK

Bola Adekoya

Nigeria

Srijana Adhikari

Nepal

Rupesh Agrawal

Singapore

Zubair M. Ahmed

USA

Mehmet Orcun Akdemir

Turkey

Sibel Aksoy

Turkey

Luciana Alencar

Brazil

Mohammad Ali

India

Jorge Alio

Spain

Micol Alkabes

Italy

\author{
Ryham Allam \\ Egypt \\ Benjamin Althouse \\ USA \\ Winfried Amoaku \\ UK \\ Afsaneh Amouzegar \\ USA
}

Kumarasamy Anbarasu

India

Marcus Ang

Singapore

Rajendra Apte

USA

James Aquavella

USA

Maria Cecilia Aquino

USA

Ilir Arapi

Italy

Yonca Arat

Turkey

Samuel Arba-Mosquera

Spain

Luis Arias

Spain
Murali Ariga
India

Mary Beth Aronow

USA

Hemamalini Arvind

Australia

Penny Asbell

USA

George Asimellis

USA

Ioannis Aslanides

Greece

Rashima Asokan

India

Marcel Avila

Colombia

Ahmed Awadein

Egypt

Marcelo Ayala

Sweden

Kalpana Babu Murthy

India

Simon Backhouse

Australia

Jeong Hun Bae

South Korea

Correspondence: anna.clark@biomedcentral.com

BioMed Central, Floor 6, 236 Gray's Inn Road, London WC1X 8HB, UK 
Haiqing Bai

China

Konstantinos Balaskas

UK

Brendan Barrett

UK

Robert Barry

UK

Daniel Barthelmes

Switzerland

Keith Barton

UK

Ken Bassett

Canada

Roberto Bellucci

Italy

Pooja Bhat

USA

Muna Bhende

India

Marc Biarnés

Spain

Catherine Birt

USA

Jenefer Blackwell

Australia

Maria Antonietta Blasi

Italy

ostas Boboridis

Greece

Kathryn Bollinger

USA

Vincenza Bonfiglio

Italy

Nacim Bouheraoua

France

Banu Bozkurt

Turkey

John Bradbury

UK

Christopher Brand

UK

Milam Brantley

USA
Fion Bremner

UK

Dominique Bremond-Gignac

France

Scott Brodie

USA

Paolo Brusini

Italy

Vatinee Bunya

USA

Kathryn Burdon

Australia

Suk Ho Byeon

South Korea

Florence Cabot

USA

Delia Cabrera Debuc

USA

Pilar Cacho-Martínez

Spain

Halil Çagatay

Turkey

Pilar Calvo

Spain

Charles Campbell

USA

Gonzalo Carracedo

Spain

Ester Carreño

UK

Pilar Casas-Llera

Spain

Laure Caspers

Belgium

Heidi Cate

UK

Lingping Cen

China

Subhabrata Chakrabarti

India

Patricia Chalela

USA

Spyridon Chalkiadakis

UK
Jagdish Chander

India

Jessica Chang

USA

Shu-Hong Chang

USA

Neil Charman

UK

William Charman

UK

Sunil Chauhan

USA

Celia Chen

Australia

Chian-Feng Chen

Taiwan

Haoyu Chen

China

Hsin-Yi Chen

Taiwan

Philip Chen

USA

Connie Chen

USA

Andy Cheng

Hong Kong

Wah Cheuk

Hong Kong

Carol Cheung

Singapore

Shravan Chintala

USA

Lidia Chomicz

Poland

Kam-Lung Kelvin Chong

Hong Kong

Nikhil Choudhari

India

Bonnie Nga Kwan Choy

Hong Kong

cqueline Chua

Singapore

Jin Kwon Chung

South Korea 
Tae-Young Chung

South Korea

Gerry Clare

UK

Rosa M. Coco

Spain

Cris Constantinescu

UK

Maria Cordeiro

UK

Luis Cordoves

Spain

Terry Coursey

USA

Margaux Crabtree

USA

Jennifer Craig

New Zealand

Ian Cree

UK

J. Oscar Croxatto

Argentina

Yan Cui

China

Arthur Cummings

Ireland

Tanuj Dada

India

Erika Damato

UK

Vivek Dave

India

Helen Davis

UK

Janet Davis

USA

Roberto Dell'Omo

Italy

Hakan Demirci

USA

Alastair Denniston

UK

Maria Dettoraki

Greece
Christoph Deuter

Germany

Federico Di Matteo

Italy

Vasilios Diakonis

USA

Xiaoyan Ding

China

Ali Djalilian

USA

Rosa Dolz-Marco

Spain

Rodrigo Donoso

Chile

Stella Douma

Greece

Laura Downie

Australia

Alina Dumitrescu

USA

Yusuf Durlu

Turkey

Matthew Edmunds

UK

Rita Ehrlich

Israel

Amal Elbendary

Egypt

Mostafa Elgohary

UK

Hala Elhilali

Egypt

Charles Elmaraghy

USA

Gehad Elnahri

Egypt

Rasha Eltanamly

Egypt

Gabriela Espinoza

USA

Boniface Ikenna Eze

Nigeria

Mads Krüger Falk

Denmark
Sepehr Feizi

Iran

Maryam Ferdousi

UK

Karen D. Fern

USA

Merle Fernandes

India

Roberto Fernandez Buenaga Spain

Giulio Ferrari

Italy

Tiago Ferreira

Portugal

Alistair Fielder

UK

Michele Figus

Italy

Robert Finger

Australia

John Flatter

USA

Ignacio Flores-Moreno

Spain

Paolo Fogagnolo

Italy

Alex Fonollosa

Spain

Alejandro Fonollosa Calduch Spain

Clare Fraser

USA

Valeria Fu

USA

Emre Güler

Turkey

Subhash Gaddipati

USA

Roberto Gallego-Pinazo

Spain

Juan Eduardo Gallo

Argentina

Nicola Gan

Singapore 


\begin{tabular}{|c|c|c|}
\hline Alfredo Garcia-Layana & Fiona Harris & Toby Hurd \\
\hline Spain & UK & UK \\
\hline Yonathan Garfias & Jiucheng He & Michele Iester \\
\hline Mexico & USA & Italy \\
\hline Sumit Garg & Mark Heaney & Makoto Inoue \\
\hline USA & USA & Japan \\
\hline David Gaucher & Anders Heijl & Makoto Ishikawa \\
\hline France & Sweden & Japan \\
\hline Bruce Gaynes & Francisco Hernandez-Torres & J. Iyer \\
\hline USA & Spain & Singapore \\
\hline Yubin Ge & Nicole Heussen & Gregory Jackson \\
\hline USA & Germany & USA \\
\hline Ronnie George & Taichi Hikichi & Robert Jacobs \\
\hline India & Japan & New Zealand \\
\hline Elham Ghahari & David Hinkle & Mohammadreza Jafarinasab \\
\hline USA & USA & Iran \\
\hline Asaad Ghanem & Nino Hirnschall & Ebrahim Jafarzadehpur \\
\hline Egypt & Austria & Iran \\
\hline Khalil Ghasemi Falavarjani & Kazuyuki Hirooka & Marilyn James \\
\hline Iran & Japan & UK \\
\hline Stephen Gichuhi & Jesper Hjortdal & Sun Young Jang \\
\hline UK & Denmark & South Korea \\
\hline Ilene Gipson & Stephan Hoffmann & Vishal Jhanji \\
\hline USA & Germany & Hong Kong \\
\hline Fleur Goezinne & Vladimir Holan & Zhengxuan Jiang \\
\hline Netherlands & Czech Republic & China \\
\hline E-Shawn Goh & Gabor Hollo & Amit Jinabhai \\
\hline Singapore & Hungary & UK \\
\hline Kong Yong Goh & Shigeru Honda & Jasleen Jolly \\
\hline Singapore & Japan & UK \\
\hline Francisco Javier Goñi & Shinataro Horie & Denis Jusufbegovic \\
\hline Spain & Japan & USA \\
\hline Johannes Gonnermann & Anna Horwood & Andreas K. Lauer \\
\hline Germany & UK & USA \\
\hline Michael Grentzelos & Akihiko Hoshi & Namir Kafil-Hussain \\
\hline Greece & Japan & UK \\
\hline Ahmet Kaan Gunduz & Mohsen Hosseini & Alon Kahana \\
\hline Turkey & Canada & USA \\
\hline Huanqing Guo & Yu-Chih Hou & Rim Kahloun \\
\hline Ireland & Taiwan & Tunisia \\
\hline Noopur Gupta & Yi-Hsun Huang & Swathi Kaliki \\
\hline India & Taiwan & India \\
\hline Sang Beom Han & Ruth Huna-Baron & Yogita Kanan \\
\hline South Korea & Israel & USA \\
\hline
\end{tabular}


Hyong Kwon Kang

Australia

Hae Min Kang

South Korea

Chitra Kannabiran

India

Costas H. Karabatsas

Greece

Dimitrios Kardaras

Greece

Marzieh Katibeh

Iran

Andreas Katsanos

Greece

S Kavitha

India

Takahiro Kawaji

Japan

Oonuma Kazuhiko

Japan

Vladimir Kefalov

USA

Hamid Khakshoor

Iran

Mayuri Khamar

India

Jane Khan

Australia

Hemant Khanna

USA

Rohit Khanna

India

Nora Khatib

USA

Ahmad Kheirkhah

USA

Kyeong Hwan Kim

South Korea

Chan Yun Kim

South Korea

Tae Wan Kim

South Korea

Kenichi Kimoto

Japan
Ashwini Kini

India

Aman Kirmani

UK

Tomas Knapen

Netherlands

Konrad Koch

Germany

Triantafyllia Koletsa

Greece

Nataliya Kolosova

Russia

Georgios Kontadakis

Greece

Karanjit Kooner

USA

Dara Koozekanani

USA

Larry Koreen

USA

Rhonda G. Kost

USA

Mihir Kothari

India

Prabhakar Krishnacharya

India

David Krizaj

USA

KazuyUKi Kumagai

Japan

Vinod Kumar

India

Periyasamy Kumar

UK

Ajay Kumar

USA

James Kundart

USA

Shoji Kuriyama

Japan

Chiara La Morgia

Italy

Neil Lagali

Sweden
Jimmy Lai

Hong Kong

Timothy Lai

Hong Kong

Cedric Lamirel

France

Jonathan Lass

USA

Alex Lau

Singapore

Augustinus Laude

Singapore

Chang Kyu Lee

South Korea

Helena Lee

UK

Salomé Leibundgut

Switzerland

Gary Joseph Lelli

USA

Gary Lelli, Jr.

USA

Michael Lemp

USA

Kim Son Lett

UK

Richard Alan Lewis

USA

Monique Leys

USA

Qingfeng Liang

China

Xiaofeng Lin

China

Gopal Lingam

Singapore

Petra Liskova

Czech Republic

Julie-Anne Little

UK

Yongji Liu

China

Bingqian Liu

China 
Wenzhong Liu

USA

Norberto Lopez-Gil

Spain

Yi Lu

China

Kenneth Lu

USA

Fiona Luk

Hong Kong

Jose Domingo Luna

Argentina

Li Ma

Hong Kong

Michele Madigan

USA

Dimitra Makrynioti

Greece

Alex Mammen

USA

Raffaele Mancino

Italy

Baskaran Mani

Singapore

Gianluca Manni

Italy

Ahmad Mansour

Lebanon

Kaweh Mansouri

Switzerland

Sanjay Marasini

New Zealand

Robert Marie-Claude

Canada

Inês Marques

Portugal

Marvin Marti

Switzerland

Enrico Martini

Italy

Vicente Martin-Montañez

Spain

Sundari Mase

USA
Ioannis Mavrikakis

UK

Julie Mcclelland

UK

Timothy Mcculley

USA

Paul Mcdaniel

USA

Rebecca Mcgreal

USA

Martin Mckibbin

UK

Rebecca Mclean

UK

Hemal Mehta

USA

Balraj Menon

USA

Farhan Merali

USA

Shannath Merbs

USA

Catherine Meyerle

USA

Manuele Michelessi

Italy

Atsuya Miki

Japan

Giovanni Milano

Italy

Dan Milea

France

Baruch Minke

Israel

Vikas Mittal

India

Garima Mittal

India

Sandra Mmontezuma

USA

Rahul Modak

India

Shaheeda Mohamed

Hong Kong
Aditi Mohla

UK

Gregory Moloney

Australia

Lieve Moons

Belgium

Bruce Moore

USA

Eduardo Moragrega

Mexico

Jiten Morarji

UK

Javier Moreno-Montañes

Spain

Nigel Morlet

Australia

Jim Morton

New Zealand

Majid Moshirfar

USA

Majid Moshirfar

USA

Lisha Mou

China

Guoying Mu

Chile

Kaustubh Mulay

India

Kosta Mumcuoglu

Israel

DaisUKe Muramatsu

Japan

Hiroshi Murata

Japan

Ian Murdoch

UK

Hemanth Murthy

India

K. Naeser

Denmark

Mayuresh Naik

India

Akira Nakai

Japan 


\begin{tabular}{|c|c|c|}
\hline $\begin{array}{l}\text { Masatsugu Nakamura } \\
\text { Japan }\end{array}$ & $\begin{array}{l}\text { Toshihiko Ohta } \\
\text { Japan }\end{array}$ & $\begin{array}{l}\text { Michael Pavlica } \\
\text { USA }\end{array}$ \\
\hline $\begin{array}{l}\text { HiroyUKi Namba } \\
\text { Japan }\end{array}$ & $\begin{array}{l}\text { Akio Oishi } \\
\text { Japan }\end{array}$ & $\begin{array}{l}\text { Gary Peh } \\
\text { Singapore }\end{array}$ \\
\hline $\begin{array}{l}\text { Mayank Nanavaty } \\
\text { UK }\end{array}$ & $\begin{array}{l}\text { Andre Okanobo } \\
\text { Brazil }\end{array}$ & $\begin{array}{l}\text { Enrico Peiretti } \\
\text { Italy }\end{array}$ \\
\hline $\begin{array}{l}\text { Raja Narayanan } \\
\text { India }\end{array}$ & $\begin{array}{l}\text { Ólöf Olafsdottir } \\
\text { Iceland }\end{array}$ & $\begin{array}{l}\text { Gökhan Pekel } \\
\text { Turkey }\end{array}$ \\
\hline $\begin{array}{l}\text { Masood Naseripour } \\
\text { Iran }\end{array}$ & $\begin{array}{l}\text { J. Nwando Olayiwola } \\
\text { USA }\end{array}$ & $\begin{array}{l}\text { Prakash Peralam Yegneswaran } \\
\text { India }\end{array}$ \\
\hline $\begin{array}{l}\text { Neema Nayeb-Hashemi } \\
\text { USA }\end{array}$ & $\begin{array}{l}\text { UchechUKwu Osuagwu } \\
\text { Australia }\end{array}$ & $\begin{array}{l}\text { Pablo Pérez-Merino } \\
\text { Spain }\end{array}$ \\
\hline $\begin{array}{l}\text { Paula Anne Newman-Casey } \\
\text { USA }\end{array}$ & $\begin{array}{l}\text { Yoko Ozawa } \\
\text { Japan }\end{array}$ & $\begin{array}{l}\text { Henry Perry } \\
\text { USA }\end{array}$ \\
\hline $\begin{array}{l}\text { Angeline Nguyen } \\
\text { USA }\end{array}$ & $\begin{array}{l}\text { Engin Bilge Ozgurhan } \\
\text { Turkey }\end{array}$ & $\begin{array}{l}\text { Simon Petersen-Jones } \\
\text { USA }\end{array}$ \\
\hline $\begin{array}{l}\text { Simona Delia Nicoara } \\
\text { Romania }\end{array}$ & $\begin{array}{l}\text { Mark Packer } \\
\text { USA }\end{array}$ & $\begin{array}{l}\text { John Phillips } \\
\text { New Zealand }\end{array}$ \\
\hline $\begin{array}{l}\text { Juan Carlos Nieto } \\
\text { Spain }\end{array}$ & $\begin{array}{l}\text { Howard Palte } \\
\text { USA }\end{array}$ & $\begin{array}{l}\text { Francesco Pichi } \\
\text { Italy }\end{array}$ \\
\hline $\begin{array}{l}\text { Kanwal Nischal } \\
\text { USA }\end{array}$ & $\begin{array}{l}\text { Chen-Wei Pan } \\
\text { China }\end{array}$ & $\begin{array}{l}\text { Jose Pinto } \\
\text { Argentina }\end{array}$ \\
\hline $\begin{array}{l}\text { Jason Noble } \\
\text { Canada }\end{array}$ & $\begin{array}{l}\text { Eleni Papageorgiou } \\
\text { UK }\end{array}$ & $\begin{array}{l}\text { Dilara Pirhan } \\
\text { Turkey }\end{array}$ \\
\hline $\begin{array}{l}\text { Benjamín Nogueda-Torres } \\
\text { Mexico }\end{array}$ & $\begin{array}{l}\text { George Papaliodis } \\
\text { USA }\end{array}$ & $\begin{array}{l}\text { Amir Pirouzian } \\
\text { USA }\end{array}$ \\
\hline $\begin{array}{l}\text { Monisha Esther Nongpiur } \\
\text { Singapore }\end{array}$ & $\begin{array}{l}\text { Chan Kee Park } \\
\text { South Korea }\end{array}$ & $\begin{array}{l}\text { Brigitte Pittet-Cuenod } \\
\text { Switzerland }\end{array}$ \\
\hline $\begin{array}{l}\text { Jean Jacques Noubiap } \\
\text { Cameroon }\end{array}$ & $\begin{array}{l}\text { Choul Yong Park } \\
\text { South Korea }\end{array}$ & $\begin{array}{l}\text { Ana Belen Plaza } \\
\text { Spain }\end{array}$ \\
\hline $\begin{array}{l}\text { Katarzyna Nowomiejska } \\
\text { Poland }\end{array}$ & $\begin{array}{l}\text { Sung Who Park } \\
\text { South Korea }\end{array}$ & $\begin{array}{l}\text { Eugenie Poh } \\
\text { Singapore }\end{array}$ \\
\hline $\begin{array}{l}\text { Carlo Nucci } \\
\text { Italy }\end{array}$ & $\begin{array}{l}\text { Katrina Parker } \\
\text { USA }\end{array}$ & $\begin{array}{l}\text { Lalitha Prajna } \\
\text { India }\end{array}$ \\
\hline $\begin{array}{l}\text { William Nunery } \\
\text { USA }\end{array}$ & $\begin{array}{l}\text { Salvador Pastor-Idoate } \\
\text { Spain }\end{array}$ & $\begin{array}{l}\text { Gaurav Prakash } \\
\text { United Arab Emirates }\end{array}$ \\
\hline $\begin{array}{l}\text { Jacqueline Nuysink } \\
\text { Netherlands }\end{array}$ & $\begin{array}{l}\text { Sangita Patel } \\
\text { USA }\end{array}$ & $\begin{array}{l}\text { Frank Proudlock } \\
\text { UK }\end{array}$ \\
\hline $\begin{array}{l}\text { Anna O'Connor } \\
\text { UK }\end{array}$ & $\begin{array}{l}\text { Vanita Pathak Ray } \\
\text { India }\end{array}$ & $\begin{array}{l}\text { Claudio Punzo } \\
\text { USA }\end{array}$ \\
\hline $\begin{array}{l}\text { Veronica O'Dwyer } \\
\text { Ireland }\end{array}$ & $\begin{array}{l}\text { Ushasree Pattamatta } \\
\text { Australia }\end{array}$ & $\begin{array}{l}\text { Dania Qatarneh } \\
\text { Australia }\end{array}$ \\
\hline $\begin{array}{l}\text { Joo Youn Oh } \\
\text { South Korea }\end{array}$ & $\begin{array}{l}\text { Jayter Paula } \\
\text { Brazil }\end{array}$ & $\begin{array}{l}\text { Hong Qi } \\
\text { China }\end{array}$ \\
\hline
\end{tabular}




\begin{tabular}{|c|c|c|}
\hline Kunliang Qiu & Andri Riau & Srinivasan Sanjay \\
\hline China & Singapore & Singapore \\
\hline Mary Qiu & Lucas Ricci & Muthupandian Saravanan \\
\hline USA & Brazil & Ethiopia \\
\hline Luciano Quaranta & Ivano Riva & Giacomo Savini \\
\hline Italy & Italy & Italy \\
\hline Francesco Quaranta Leoni & Syed Rizvi & Ariel Schlaen \\
\hline Italy & India & Spain \\
\hline Mansur Rabiu & Gloria Roberti & Leopold Schmetterer \\
\hline Nigeria & Italy & Austria \\
\hline Mohammad Taher Rajabi & Daniel Roberts & Katrina Schmid \\
\hline Iran & USA & Australia \\
\hline Rajesh Rajagopalan & Shisong Rong & Heather Schmitt \\
\hline Singapore & Hong Kong & USA \\
\hline Raju Rajala & Maged Roshdy & Lynn Schoenfield \\
\hline USA & Egypt & USA \\
\hline Vasant Raman & Luca Rossetti & Stephen Schwartz \\
\hline UK & Italy & USA \\
\hline Aparna Ramasubramanian & Avik Roy & Edina Schweighoffer \\
\hline USA & India & UK \\
\hline Pradeep Ramulu & Sanhita Roy & Leonard Seibold \\
\hline USA & India & USA \\
\hline Padmaja Kumari Rani & Sayon Roy & Sirisha Senthil \\
\hline India & USA & India \\
\hline Aparna Rao & Jos Rozema & Yasir Sepah \\
\hline India & Belgium & USA \\
\hline Dejan M. Rašić & Eliana Rulli & Robert Sergott \\
\hline Serbia & Italy & USA \\
\hline Rinki Ratnapriya & Kai Saariniemi & Ameet Shah \\
\hline USA & Finland & UK \\
\hline Kimberly Reed & li Osman Saatci & Syed Shah \\
\hline USA & Turkey & USA \\
\hline Cornelius Rene & Sergio Saccà & Zaid Shalchi \\
\hline UK & Italy & UK \\
\hline Lixing Reneker & Mahipal Sachdev & Siddesh Shambhu \\
\hline USA & India & UK \\
\hline Joanna Reszeć & Mohammad Sadiq & Robert Shanks \\
\hline Poland & USA & USA \\
\hline Amanda Rey & Manuel Saenz-De-Viteri & S. Shantha \\
\hline Spain & Spain & India \\
\hline Flavio Rezende & Masaaki Saito & Emily Shao \\
\hline Canada & Japan & UK \\
\hline Seungsoo Rho & Pavandeep Singh Sandhu & Heather Sheardown \\
\hline South Korea & UK & Canada \\
\hline
\end{tabular}


Sethu Sheeladevi

India

Raneen Shehadeh Mashor Israel

Yin Shen

China

Haihong Shi

China

Tomoaki Shiba

Japan

Rebecca Shields

USA

Rabia Shihada

Israel

Katsunori Shimada

Japan

Ari Shinojima

Japan

Jyoti Shrestha

Nepal

Ho Cheung (Anderson ) Shum

Hong Kong

Damian Siedlecki

Poland

Eric Sigler

USA

Ronald H. Silverman

USA

Huseyin Simavli

Turkey

Matthew Simunovic

UK

Michael Singer

USA

Eric Singman

USA

Sobha Sivaprasad

UK

Simon Skalicky

UK

Dimitra Skondra

USA

Wendy M Smith

USA
John Somner

UK

Kaz Soong

USA

Luigina Sorbara

Canada

Leopoldo Spadea

Italy

Oriel Spierer

Israel

Alex Spratt

USA

Sandra Spurr-Michaud

USA

Magi Sque

UK

Prema Sriram

Australia

Erin Stahl

USA

Miles Stanford

UK

David Steel

UK

John Stephenson

UK

Jay Stewart

USA

Gregory Stoddard

USA

Rupert Wolfgang Strauss

USA

Veit Sturm

Switzerland

Kaiming Su

China

Guanfang Su

China

Lakshman Subbaraman

Canada

Aditya Sudhalkar

India

Alan Sugar

USA
Michelle Sun

Australia

Young Sun

USA

Shivalingappa Swamynathan

USA

Mark Swanson

USA

Celine Sys

UK

Christopher Ta

USA

Audrey Talley Rostov

USA

Petrina Tan

Singapore

NaoyUKi Tanimoto

Germany

Angelo P. Tanna

USA

Jeremiah Tao

USA

Andrew Taylor

USA

Alejandro Tello

Colombia

Stephen Teoh

Singapore

Miguel Teus

Spain

Margarita Todorova

Switzerland

Mustafa Toker

Turkey

Ebru Toker

Turkey

Javier Tomás-Juan

Spain

Antonio Toso

Italy

Yuksel Totan

Turkey

Sharon Tow

Singapore 
Chieh-Chih Tsai

Taiwan

Yeou-Ping Tsao

Taiwan

Michael Tsatsos

UK

Kiran Turaka

USA

Fatih Turkcu

Turkey

Phillip Turnbull

New Zealand

Argyrios Tzamalis

Greece

Takashi Ueta

Japan

F. Ussa

USA

Alejandra Valenzuela

USA

Tom Van Den Berg

Netherlands

Suzanne Van Landingham

USA

Gregory Van Stavern

USA

Lourens Van Zyl

Australia

Jean Vaudaux

Switzerland

Demetrios Vavvas

USA

Ramesh Ve

India

Federico Velez

USA

Rengaraj Venkatesh

India

Lingam Vijaya

India

Edoardo Villani

Italy

Guadalupe Villarreal Jr.

USA
Catherine Viner

UK

Gianni Virgili

Italy

Michael Volkert

USA

Graham Wallace

UK

Yan Wang

China

I-Jong Wang

Taiwan

An-Guor Wang

Taiwan

Gaofeng Wang

USA

Mengmeng Wang

China

Zhongxiao Wang

USA

Yi Wei

USA

Derek Welsbie

USA

Adam Wenick

USA

Eric Wieben

USA

Helmut Wilhelm

Germany

Cathy Williams

UK

David Williams

UK

Pete Williams

USA

Kimberly Winges

USA

Meraf Wolle

USA

Ian Wong

Hong Kong

Elizabeth Wong

Singapore
Wolf Wonneberger

Sweden

Se Joon Woo

South Korea

Emily Woodman

Australia

Fasika Woreta

USA

Yuan Wu

China

Jason CheUK-Sing Yam

Hong Kong

SUK-Woo Yang

South Korea

Chung-May Yang

Taiwan

Yit Yang

UK

Hui Yang

China

Yufeng Yao

China

Ke Yao

China

Yu-Feng Yao

China

Yasemin Yavuz

Turkey

Volkan Yaylali

Turkey

Tun K Yeo

Singapore

Zheng Qin Yin

China

Efdal Yoeruek

Germany

David Yoo

USA

Kyung Chul Yoon

South Korea

Sam Young Yoon

South Korea

Kim Yoon-Duck

South Korea 
Yosanan Yospaiboon

Thailand

Honghua Yu

China

Minbin Yu

China

Murat Yuksel

Turkey

Patrick Yu-Wai-Man

UK

Leandro Zacharias

Brazil

Masahiro Zako

Japan
Juan Carlos Zenteno

Mexico

Xiaojun Zhang

China

Xiongze Zhang

China

Yuhua Zhang

USA

Shaoru Zhang

China

Xiulan Zhang

China

Chengxin Zhou

USA
Shiyou Zhou

China

Meidong Zhu

Australia

Mohammed Ziaei

UK

Noel Ziebarth

USA

Haidong Zou

China 